\title{
Nephroprotective Effect of Coenzyme Q10 alone and in Combination with $\mathrm{N}$-acetylcysteine in Diabetic Nephropathy
}

Shri Neminath Jain Brahmacharyashram Trust's

Shreeman Sureshdada Jain College of Pharmacy

Nashik, Maharashtra, India

Received 3 November, 2020, accepted 29 March, 2021

Abstract Aim: Oxidative stress due to chronic hyperglycaemia is a key factor in the development and progression of various microvascular complications including diabetic nephropathy (DN) and associated renal injury. Treatment with antioxidants is one of the strategies to protect the kidney from oxidative tissue damage to improve renal physiology during DN. The investigation, therefore, was designed to assess the nephroprotective effect of coenzyme Q10 (CoQ10) and N-acetylcysteine (NAC), either alone or in combination in streptozotocin (STZ)-nicotinamide (NAD) induced diabetic nephropathy (DN) in rats.

Methods: T2DM induced by STZ (55 mg/kg, i.p.)-NAD (110 mg/kg, i.p.) in Sprague-Dawley rats $(220-250 \mathrm{~g})$ was confirmed by the elevated blood glucose level and glycated haemoglobin. DN was assessed by renal function tests. The diabetic rats were treated with CoQ10 (10 mg/kg, p.o.) and/or NAC (300 mg/kg, p.o.) for 8 weeks after confirmation of DN. Oxidative tissue damage due to STZ-NAD was estimated by malondialdehyde (MDA), superoxide dismutase (SOD) and catalase (CAT), reduced glutathione (GSH), myeloperoxidase (MPO) and nitric oxide (NO) in the renal homogenate.

Results: Data showed significant alteration in serum and urinary creatinine, total protein, albumin, serum urea, blood urea nitrogen (BUN) and uric acid in diabetic animals as compared to the control rats. CoQ10 and/or NAC effectively alleviated the disturbances in renal function. Diabetic rats showed increased MDA, decreased SOD and CAT activities and decreased GSH along with a significant increase in MPO activity and nitrite content. Treatment with the aforementioned antioxidants and their combination ameliorated the kidney damage as indicated by the reduced OS with improved renal function.

Conclusion: The investigation suggests that the chronic hyperglycaemia-induced OS leads to the development and progression of DN. The combined treatment with $\mathrm{CoQ} 10$ and NAC has shown a remarkable nephroprotective effect suggesting that combined antioxidant therapy with CoQ10 and NAC may be useful in the attenuation of DN.

Keywords Diabetic nephropathy-oxidative stress - streptozotocin-coenzyme Q10-N-acetylcysteine

\section{INTRODUCTION}

Diabetes mellitus (DM) is a group of heterogeneous disorders characterized by hyperglycaemia linked to insulin deficiency or decreased tissue sensitivity (Maitra, 2015; Masharani, 2008). The two types of DM are type 1 DM (T1DM) and type 2 DM (T2DM).

T2DM or non-insulin-dependent diabetes mellitus (NIDDM), a multifactorial disorder develops as a result of insulin resistance with relative insulin deficiency and is most common among adults. T2DM features a strong genetic component in addition to the acquired pathogenic causes associated with secretion and resistance to insulin (Kohei, 2010).

Premature deaths due to DM are associated with macro and microvascular complications. The primary microvascular complications of DM are diabetic nephropathy (DN), diabetic neuropathy and diabetic retinopathy (Pal et al., 2014; Geraldes et al., 2009).

DN is the foremost cause of chronic kidney disease (CKD) and end-stage renal disease (ESRD). It is a progressive and irreversible loss of renal function involving glomerular hyperfiltration, incipient nephropathy, microalbuminuria, overt proteinuria and ESRD (Mogensen et al., 1983). DN induces renal structural changes like an accumulation of extracellular matrix and glomerular mesangial expansion, interstitial fibrosis and basement membrane thickening (Vora \& Ibrahim, 2003).

Hyperglycaemia plays a crucial role in the development of DN. It has been shown that persistent hyperglycaemia in DM can induce OS by several mechanisms that involve, glucose auto- 
oxidation, activation and acceleration of polyol pathway, protein glycation through non-enzymatic means and reduced antioxidant defense. Hyperglycaemia is believed to act by activation of Protein kinase C (PKC) pathway, generation of reactive oxygen species (ROS) and over-expression of transforming growth factor- $\beta$ (TGF- $\beta$ ) (Schena \& Gesualdo, 2005; Brownlee, 2001).

The normal kidney owing to its high metabolic activity generates substantial OS that is stabilized by an antioxidant defense system. Uncontrolled blood glucose level shifts this equilibrium to a pro-oxidant state resulting in tissue injury and vascular anomalies It has been shown that the mechanisms involved in the development of the DN induce considerable OS by one or other means (Vasavada \& Agarwal, 2005).

At present, none of the therapies used for DN have proved efficacious due to the involvement of diverse etiologic factors in the progression of DN. Therefore, it is difficult to select the best possible treatment approach and therapeutic agent. Consequently, effective and novel therapeutic strategies are required in the treatment of DN. Studies have demonstrated the beneficial effects of antioxidant therapies in DN (Mahajan et al., 2019).

CoQ10 (ubiquinone) is an endogenous, vitamin-like lipophilic substance serving as a natural antioxidant. CoQ10 plays a key role in the mitochondrial electron transport chain and scavenges free radicals to protect the $\beta$ cells from the harmful effects of OS by its antioxidant properties (Hodgson et al., 2002; Rosenfeldt et al., 2007). Studies have also shown that CoQ10 is effective for glycaemic control among individuals with T2DM and reduces $\mathrm{HbA}_{1 c}$ levels (Maheshwari et al., 2014). NAC is a glutathione precursor and a powerful antioxidant. NAC has been shown to impart protection to the $\beta$-cells against cellular damage. NAC attenuates hyperglycaemia and improves glucose intolerance. This action of NAC is linked to glucose-induced insulin release (Haber et al., 2003; Ahmad et al., 2012; Shimizu et al., 2005).

So far, no earlier studies have reported the synergistic effect of CoQ10 and NAC in the treatment of DN. Hence, we have selected these antioxidants and an effort was taken to study their combined effect in nephroprotection against experimentally induced DN. Therefore, the current research was aimed to explore the nephroprotective effect of CoQ10 and NAC alone and in combination in STZ-NAD-induced DN.

\section{METHODS}

\section{Drugs and Chemicals}

CoQ10 was obtained as a gift sample from Zydus Cadila, Ahmedabad, India. NAC was purchased from Loba Chemie (Mumbai, India). STZ and NAD were purchased from SigmaAldrich (USA). Spectrophotometric kits for determination of superoxide dismutase (SOD), catalase (CAT), and myeloperoxidase (MPO) activity and malondialdehyde (MDA), reduced glutathione (GSH) and nitric oxide (NO) content were acquired from Elabscience Biotechnology Inc. (Houston, USA). Kits for estimation of total protein and albumin were obtained from Arkray Healthcare Pvt. Ltd. (Mumbai, India). Determination of serum and urinary creatinine, serum urea, BUN and uric acid was carried by using the biochemical kits purchased from Tulip Diagnostics Pvt. Ltd. (Mumbai, India). All the other chemicals and reagents used in the study were of analytical grade.

\section{Animals}

Forty healthy male Sprague-Dawley rats (SD, 8 weeks, 220-250 g) were obtained from the National Institute of Bioscience, Pune. Rats were acclimated for 1 week before the commencement of the study. Animals were housed under a controlled environment of temperature $\left(18-22^{\circ} \mathrm{C}\right)$ and light (12 hr light/dark cycle, lights on 07:00-19:00). All the rats were provided ad libitum access to water and standard food. All the experimental procedures in this study were carried out following the guidelines issued by the Committee for the Purpose of Control and Supervision of Experiments on Animals (CPCSEA) for the care and use of animals. The Institutional Animal Ethics Committee (IAEC) approved the protocol of the study bearing the reference number SSDJ/ IAEC/2016/02.

\section{Induction of Diabetic Nephropathy}

T2DM was induced in overnight fasted male SD rats using STZ (55 mg/kg) as a single intraperitoneal (i.p.) injection. STZ was dissolved in freshly prepared cold citrate buffer $(\mathrm{pH} 4.5)$ and administered immediately. STZ was injected $15 \mathrm{~min}$. after the administration of nicotinamide $(110 \mathrm{mg} / \mathrm{kg}$, i.p.) dissolved in normal saline (Ghasemi et al., 2014; Badole et al., 2013). Hyperglycaemia was determined at $72 \mathrm{~h}$ and then at the end of the first week post-treatment with STZ-NAD using a glucometer (AlereG1, Korea). Blood glucose level was estimated in the samples collected from the end part of tails. Animals showing elevated blood glucose level greater than $250 \mathrm{mg} / \mathrm{dL}$ were served as diabetic and were included in the nephropathy studies.

\section{Experimental Design}

Rats were divided into the following five groups with six animals each.

Group I: Normal rats (control, negative control without any treatment)

Group II: Diabetic control rats (STZ-NAD DN, positive control without any treatment)

Group III: Diabetic rats + CoQ10 (10 mg/kg/day) suspended in $1 \%$ aqueous solution of Tween 80 and designated as CoQ10 group (Garjani et al., 2011; Maheshwari et al., 2014) 
Group IV: Diabetic rats + NAC (300 mg/kg/day) dissolved in distilled water and designated as NAC group (Lee et al., 2016; Odetti et al., 2003)

Group V: Diabetic rats + CoQ10 and NAC (CoQ10 + NAC group) CoQ10 and NAC were administered orally to rats using an intragastric tube daily for a period of 8 weeks.

Treatment of Diabetic rats from groups III, IV and V was initiated at the beginning of the 5th week and continued till the end of the 12th week of the study duration.

At the end of the experimental period, and twenty-four hours after the last antioxidant dose, the rats were placed individually in the metabolic cages for 24 hours to collect urine samples. The urine samples were centrifuged and stored in ambercoloured vials. After that, blood samples from retro-orbital plexus were collected from the rats under pentobarbitone sodium anaesthesia. Whole blood collected in commercially available ethylene diamine tetra-acetic acid (EDTA) tubes was used for the estimation of HbA1c. Serum was separated by allowing the collected blood to clot in tubes without EDTA and was used for the estimation of renal parameters. Both urine and serum samples used for biochemical assessment were stored at $-20^{\circ} \mathrm{C}$.

$\mathrm{HbA}_{1 \mathrm{c}}$ was estimated from whole blood samples. Total protein, albumin and creatinine were estimated in serum and urine samples. Urea, BUN and uric acid were determined from serum with the help of biochemical kits using Prietest Touch Biochemistry Analyzer, Robonik India Pvt. Ltd. (Mumbai, India).

Creatinine clearance $(\mathrm{Ccr}, \mathrm{mL} / \mathrm{min})$ was determined from the following formula: (urine creatinine $[\mathrm{mg} / \mathrm{dL}] \times 24 \mathrm{~h}$ urine volume $[\mathrm{mL}]) /($ serum creatinine $[\mathrm{mg} / \mathrm{dL}] \times 1440[\mathrm{~min}])$. Urinary albumin excretion rate (UAER, $\mu \mathrm{g} / \mathrm{min}$ ) was calculated by using the formula: Albumin $[\mathrm{mg} / \mathrm{dL}] \times$ volume of urine in timed collection $[\mathrm{dL}] \times 1000) / 1440[\mathrm{~min}]$.

At the end, the rats were sacrificed by an overdose of pentobarbitone sodium ( $200 \mathrm{mg} / \mathrm{kg}$, body weight, i.p.) and the kidneys were removed, washed with ice-cold saline, weighed and processed for tissue biochemical estimations.

\section{Estimation of markers of Oxidative Stress}

The dissected kidneys were placed in a Petri plates with ice-cold conditions. The tissues were sliced using a surgical scalpel in the presence of chilled 0.25 M sucrose. These were then blotted quickly using filter paper. These were minced and homogenized with 25 strokes of tight Teflon pestle of glass homogenizer at a speed of $10,000 \times \mathrm{g}$ at $0^{\circ} \mathrm{C}$ using the Remi cooling centrifuge. Either normal saline or phosphatebuffered saline (PBS) was used as the homogenization medium. $10 \% \mathrm{w} / \mathrm{v}$ tissue homogenate was prepared according to the protocol supplied by the manufacturer with the respective antioxidant enzyme assay kit. The total protein concentration in the homogenate was determined using a total protein assay kit (Bicinchonic acid method, E-BC-K075).

\section{Statistical analysis}

All the data were expressed as mean \pm standard error of the mean (S. E. M.). One-way ANOVA was used to record intergroup variation followed by Tukey's multiple comparisons test as appropriate to test statistical significance using Graph Pad Prism version 5.0, GraphPad Software, Inc. The minimum significance level was set at $p<0.05$ for all the tests.

\section{RESULTS}

\section{Effect of CoQ10, NAC or their combination on body weight $\%$}

Significant $(p<0.05)$ difference in body weight $\%$ was observed when all the groups were compared (Table 1). Normal rats showed a significant $(p<0.05)$ increase in body weight $\%$ at the twelfth week in contrast to the STZ-NAD treated diabetic rats with a significant $(p<0.05)$ reduction in body weight $\%$ at the same period. Diabetic groups treated with CoQ10 and/or NAC prevented such a weight loss in comparison to the diabetic control group (Table 1). A significant $(p<0.05)$ effect of combined administration of CoQ10 and NAC on body weight $\%$ in diabetic rats was noted as compared to the diabetic animals receiving CoQ10 or NAC alone.

\section{Effect of CoQ10, NAC or their combination on the kidney to body weight ratio}

As shown in Table 1, induction of T2DM with STZ-NAD in the rats significantly $(p<0.05)$ increased the kidney to body weight ratio than in the normal rats. Diabetic rats treated with CoQ10, NAC or their combination (CoQ10 + NAC) for eight weeks (5th-12th week) significantly $(p<0.05)$ prevented the increase in kidney to body weight ratio as compared to the diabetic control rats. On the other hand, the combined administration of CoQ10 and NAC significantly $(p<0.05)$ abrogated the elevation in kidney to body weight ratio as compared to the diabetic control group and the diabetic rats receiving either CoQ10 or NAC alone.

\section{Effect of CoQ10, NAC or their combination on blood glucose level and $\mathrm{HbA}_{1 \mathrm{C}}$}

As given in Table 2, $72 \mathrm{~h}$ post STZ-NAD injection (week 0) showed a significant $(p<0.05)$ increase in blood glucose level of all the treatment groups as compared to the normal rats. Oral administration of CoQ10 showed a significant $(p<0.05)$ decrease in blood glucose levels relative to the diabetic animals. A significant $(p<0.05)$ decrease in blood glucose level was observed in the diabetic animals treated with NAC as compared to the diabetic control group. It was also noted that NAC didn't affect the elevated blood glucose level in comparison to the observations with NAC treated diabetic rats at week 0 (Table 2). Treatment of the diabetic rats with combination of 
Table 1. Effect of CoQ10, NAC or their combination on body weight\% and kidney to body weight ratio $(\mathrm{mg} / \mathrm{g})$.

\begin{tabular}{|c|c|c|c|}
\hline \multirow{2}{*}{ Groups } & \multicolumn{2}{|c|}{ Body weight \% } & \multirow{2}{*}{ Kidney to Body Weight Ratio (mg/g) } \\
\cline { 2 - 3 } & Initial at Week 0 & Final at Week 12 & $3.807 \pm 0.041$ \\
\hline Control & $100.0 \pm 0.368$ & $145.2 \pm 0.865^{*}$ & $7.504 \pm 0.193^{\mathrm{a}}$ \\
\hline STZ-NAD DN & $100.0 \pm 1.389$ & $87.5 \pm 0.958^{\mathrm{a}^{*}}$ & $5.490 \pm 0.199^{\mathrm{ab}}$ \\
\hline CoQ10 $(10 \mathrm{mg} / \mathrm{kg})$ & $100.0 \pm 0.763$ & $105.2 \pm 1.122^{\mathrm{ab}^{*}}$ & $5.201 \pm 0.072^{\mathrm{ab}}$ \\
\hline NAC $(300 \mathrm{mg} / \mathrm{kg})$ & $100.0 \pm 1.128$ & $106.5 \pm 0.467^{\mathrm{ab}^{*}}$ & $4.530 \pm 0.126^{\mathrm{abcd}}$ \\
\hline CoQ10 NAC & $100.0 \pm 1.039$ & $112.0 \pm 1.217^{\mathrm{abcd}^{*}}$ & \\
\hline
\end{tabular}

Values are expressed as mean $\pm S E M ; n=6$. One-way ANOVA followed by Tukey's multiple comparison test. ${ }^{a} p<0.05$ as compared to the normal control group, ${ }^{b} p<0.05$ as compared to the diabetic control group, ${ }^{c} p<0.05$ as compared to diabetic CoQ 10 alone treated group and ${ }^{d} p<0.05$ as compared to diabetic NAC alone treated group, ${ }^{*} p<0.05$ as compared with the same group at 0 week.

Table 2. Effect of CoQ10, NAC or their combination on blood glucose level ( $\mathrm{mg} / \mathrm{dL})$ and $\mathrm{Hb} \mathrm{A}_{1 \mathrm{C}}$.

\begin{tabular}{|c|c|c|c|}
\hline \multirow{2}{*}{ Groups } & \multicolumn{2}{|c|}{ Blood Glucose (mg/dL) } & \multirow{2}{*}{ HbA $_{1 \mathrm{C}}$ (\%) } \\
\cline { 2 - 3 } & Initial at Week 0 & Final at Week 12 & $4.733 \pm 0.084$ \\
\hline Control & $91.83 \pm 2.937$ & $91.0 \pm 3.13$ & $9.05 \pm 0.283^{\mathrm{a}}$ \\
\hline STZ-NAD DN & $499.0 \pm 17.27^{\mathrm{a}}$ & $534.0 \pm 24.28^{\mathrm{a}}$ & $6.15 \pm 0.080^{\mathrm{ab}}$ \\
\hline CoQ10 (10 mg/kg) & $473.2 \pm 17.35^{\mathrm{a}}$ & $413.5 \pm 5.812^{\mathrm{ab}^{*}}$ & $6.4 \pm 0.085^{\mathrm{ab}}$ \\
\hline NAC (300 mg/kg) & $480.0 \pm 15.73^{\mathrm{a}}$ & $451.7 \pm 11.8^{\mathrm{ab}}$ & $5.4 \pm 0.171^{\mathrm{abcd}}$ \\
\hline CoQ10 NAC & $477.7 \pm 15.34^{\mathrm{a}}$ & $351.5 \pm 12.03^{\mathrm{abcd}}$ & \\
\hline
\end{tabular}

Values are expressed as mean $\pm S E M ; n=6$. One-way ANOVA followed by Tukey's multiple comparison test. ${ }^{a} p<0.05$ as compared to the normal control group, ${ }^{b} p<0.05$ as compared to the diabetic control group, ${ }^{c} p<0.05$ as compared to diabetic CoQ 10 alone treated group and ${ }^{d} p<0.05$ as compared to diabetic NAC alone treated group, ${ }^{*} p<0.05$ as compared with the same group at 0 week.

CoQ10 and NAC resulted in significant $(p<0.05)$ attenuation of hyperglycaemia as compared to the other diabetic groups with or without treatment included in the study.

$\mathrm{HbA}_{1 \mathrm{c}}$ was significantly $(p<0.05)$ elevated in the diabetic control rats than the normal rats (Table 2). Treatment with CoQ10 resulted in a significant $(p<0.05)$ change in $\mathrm{HbA}_{1 c}$ as compared to the diabetic control rats. NAC treatment significantly $(p<0.05)$ decreased $\mathrm{HbA}_{1 \mathrm{c}}$ than the diabetic rats. The combination treatment decreased $\mathrm{HbA}_{1 \mathrm{c}}$ level significantly $(p<0.05)$ as compared to the diabetic groups treated with CoQ10 or NAC alone (Table 2).

Effect of CoQ10, NAC or their combination on serum total protein, albumin, creatinine and creatinine clearance

A significant $(p<0.05)$ decline in renal function was observed in the diabetic control rats than the normal rats (Table 3 ). Diabetic rats treated with CoQ10, NAC or their combination significantly $(p<0.05)$ improved the renal function than the diabetic control group. The creatinine clearance in the diabetic rats receiving the aforementioned treatments was improved significantly $(p<0.05)$ as compared to the diabetic control group. The combination treatment had a more beneficial effect on the restoration of renal function (Table 3 ).

\section{Effect of CoQ10, NAC or their combination on serum urea, BUN and uric acid}

As illustrated in Figure 1, the diabetic rats treated with CoQ10, NAC or CoQ10+NAC resulted in a significant $(p<$ 0.05 ) decrease in serum urea (Fig. 1a), BUN (Fig. 1b) and uric acid levels (Fig. 1c). More beneficial effect of the combination treatment in diabetic rats was noted in terms of significant $(p<0.05)$ difference in these parameters than the diabetic groups treated with CoQ10 or NAC alone.

Values are expressed as mean $\pm \mathrm{SEM} ; n=6$. One-way ANOVA followed by Tukey's multiple comparison test. ${ }^{a} p<0.05$ as compared to the normal control group, ${ }^{b} p<0.05$ as compared to the diabetic control group, ${ }^{c} p<0.05$ as compared to diabetic CoQ10 alone treated group and ${ }^{\mathrm{d}} p<0.05$ as compared to diabetic NAC alone treated group.

\section{Effect of CoQ10, NAC or their combination on urine volume, urinary protein and albumin excretion}

In the diabetic control group, $24 \mathrm{~h}$ urine volume (Fig. 2a) increased significantly $(p<0.05)$ than the normal rats. Diabetic groups treated with CoQ10, NAC or their combination showed a significant $(p<0.05)$ decrease in the urine output as compared to the diabetic control group without any 
Table 3. Effect of CoQ10, NAC or their combination on serum total protein, albumin, creatinine and creatinine clearance.

\begin{tabular}{|c|c|c|c|c|}
\hline Groups & $\begin{array}{c}\text { Total Protein } \\
(\mathbf{g} / \mathbf{d L})\end{array}$ & $\begin{array}{c}\text { Albumin } \\
(\mathbf{g} / \mathbf{d L})\end{array}$ & $\begin{array}{c}\text { Creatinine } \\
(\mathbf{m g} / \mathbf{d L})\end{array}$ & $\begin{array}{c}\text { Creatinine clearance } \\
(\mathbf{m L} / \mathbf{m i n})\end{array}$ \\
\hline Control & $7.55 \pm 0.071$ & $3.65 \pm 0.042$ & $0.426 \pm 0.032$ & $1.134 \pm 0.088$ \\
\hline STZ-NAD DN & $4.88 \pm 0.094^{\mathrm{a}}$ & $1.86 \pm 0.114^{\mathrm{a}}$ & $2.7 \pm 0.182^{\mathrm{a}}$ & $0.269 \pm 0.030^{\mathrm{ab}}$ \\
\hline CoQ10 $(10 \mathrm{mg} / \mathrm{kg})$ & $6.21 \pm 0.149^{\mathrm{ab}}$ & $2.95 \pm 0.117^{\mathrm{ab}}$ & $1.51 \pm 0.110^{\mathrm{ab}}$ & $0.631 \pm 0.039^{\mathrm{ab}}$ \\
\hline NAC $(300 \mathrm{mg} / \mathrm{kg})$ & $5.93 \pm 0.195^{\mathrm{ab}}$ & $2.56 \pm 0.133^{\mathrm{ab}}$ & $1.55 \pm 0.105^{\mathrm{ab}}$ & $0.608 \pm 0.045^{\mathrm{ab}}$ \\
\hline CoQ10 NAC & $6.9 \pm 0.063^{\mathrm{abcd}}$ & $3.05 \pm 0.067^{\mathrm{abd}}$ & $1.05 \pm 0.058^{\mathrm{abcd}}$ & $0.907 \pm 0.039^{\mathrm{abcd}}$ \\
\hline
\end{tabular}

Values are expressed as mean \pm SEM; $n=6$. One-way ANOVA followed by Tukey's multiple comparison test. ${ }^{a} p<0.05$ as compared to the normal control group, ${ }^{b} p<0.05$ as compared to the diabetic control group, $c p<0.05$ as compared to diabetic CoQ10 alone treated group and ${ }^{d} p<0.05$ as compared to diabetic NAC alone treated group.

treatment.

There was a significant $(p<0.05)$ elevation in the urinary protein (Fig. 2b) and UAER (Fig. 2c) of the diabetic rats than the normal rats. The treatment of diabetic groups with CoQ10, NAC or their combination significantly $(p<0.05)$ reduced the appearance of proteins in the urine and UAER as compared to the diabetic control group.

It was noted that Co-administration of CoQ10 and NAC to the diabetic rats led to a more significant effect in reducing UAER Values are expressed as mean $\pm \mathrm{SEM} ; n=6$. One-way ANOVA followed by Tukey's multiple comparison test. ${ }^{a} p<0.05$ as compared to the normal control group, ${ }^{\mathrm{b}} p<0.05$ as compared to the diabetic control group, ${ }^{c} p<0.05$ as compared to diabetic CoQ10 alone treated group and ${ }^{d} p<0.05$ as compared to diabetic NAC alone treated group.

\section{Effect of CoQ10, NAC or their combination on renal oxidative stress and anti-oxidant markers}

Diabetic control rats showed significant $(p<0.05)$ increase in the MDA. Treatment of diabetic rats using the combination of CoQ10 and NAC after induction of DN exhibited significant ( $p$ $<0.05)$ reduction in MDA than the diabetic rats treated orally with CoQ10 or NAC alone (Fig. 3a).

The activities of SOD and CAT enzymes in the kidney of diabetic control rats were significantly $(p<0.05)$ decreased. The oral administration of CoQ10, NAC or CoQ10 + NAC to the diabetic rats significantly $(p<0.05)$ restored the activities SOD and CAT (Fig. 3b \& c). The GSH level was significantly ( $p$ $<0.05$ ) decreased in STZ-NAD treated rats, as compared to the normal rats. Intragastric administration of CoQ10, NAC or CoQ10 + NAC to the diabetic rats showed significant increase $(p<0.05)$ in the GSH (Fig. 3d).

Values are expressed as mean $\pm \mathrm{SEM} ; n=6$. One-way ANOVA followed by Tukey's multiple comparison test. ${ }^{a} p<0.05$ as compared to the normal control group, ${ }^{\mathrm{b}} p<0.05$ as compared to the diabetic control group, ${ }^{c} p<0.05$ as compared to diabetic CoQ10 alone treated group and ${ }^{d} p<0.05$ as compared to diabetic NAC alone treated group. a
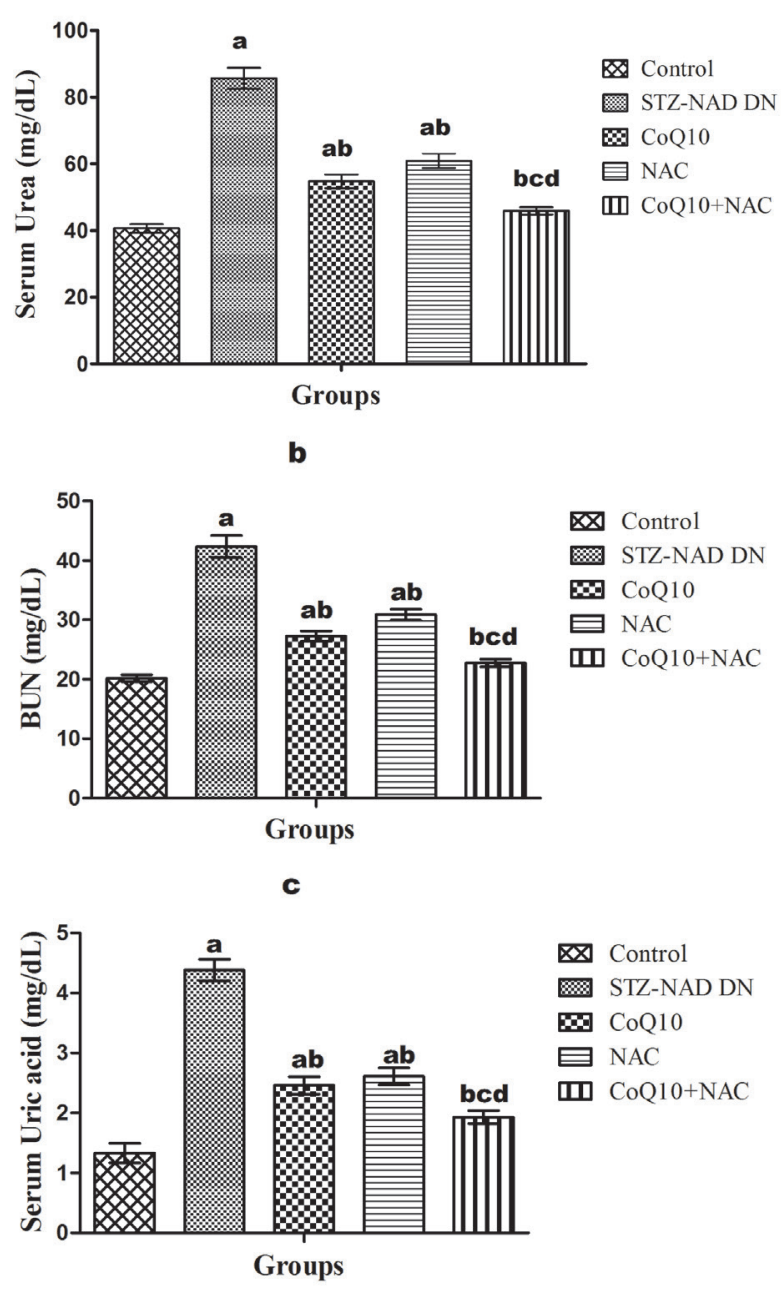

Figure 1. Effect of CoQ10, NAC or their combination on a) serum urea, b) BUN and c) uric acid. 
a

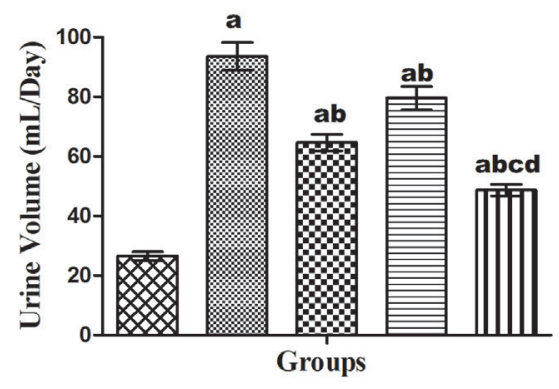

b
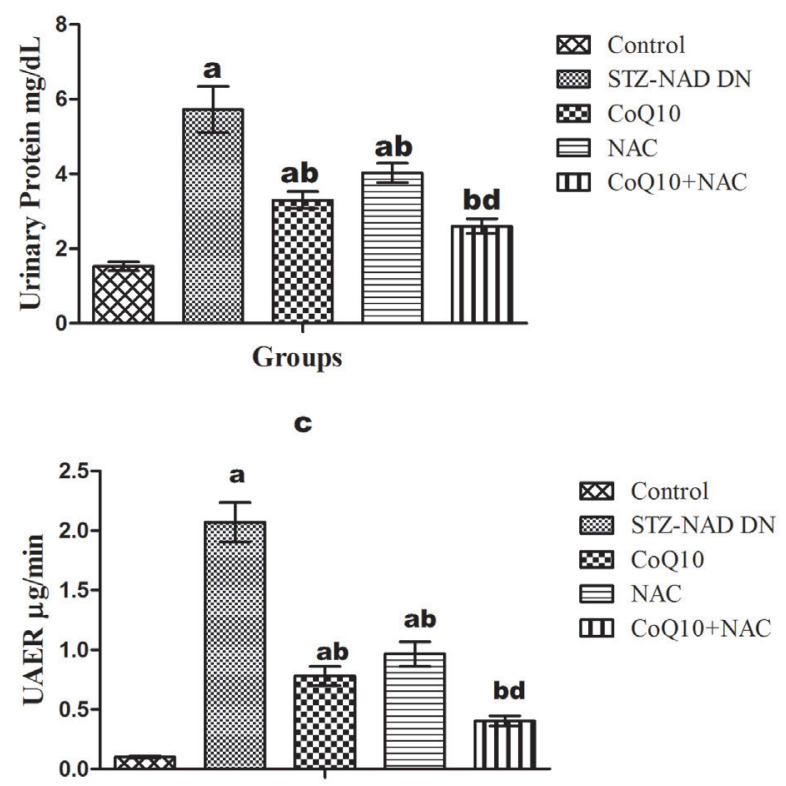

Groups

Figure 2. Effect of CoQ10, NAC or their combination on a) urine volume ( $\mathrm{mL} / \mathrm{Day})$, b) urinary protein $(\mathrm{mg} / \mathrm{dL})$ and c) UAER $(\mu \mathrm{g} / \mathrm{min})$.

\section{Effect of CoQ10, NAC or their combination on MPO activity and NO content in renal tissue}

As given in Table 4, MPO activity and nitrite content were significantly $(p<0.05)$ increased in the kidney of diabetic control rats as compared to the normal rats. CoQ10, NAC or their combination significantly $(p<0.05)$ reversed the MPO activity and nitrite. More significant $(p<0.05)$ decrease in these parameters was noted with the combined treatment of CoQ10 and NAC to the diabetic rats.

\section{DISCUSSION}

The aim of the present study was to investigate the effect of CoQ10 and NAC alone and in combination on STZ-NAD induced rat model of DN. In the current investigation, STZNAD administration to rats resulted in classical features of DM, like hyperglycaemia, retarded growth, polyuria, proteinuria,
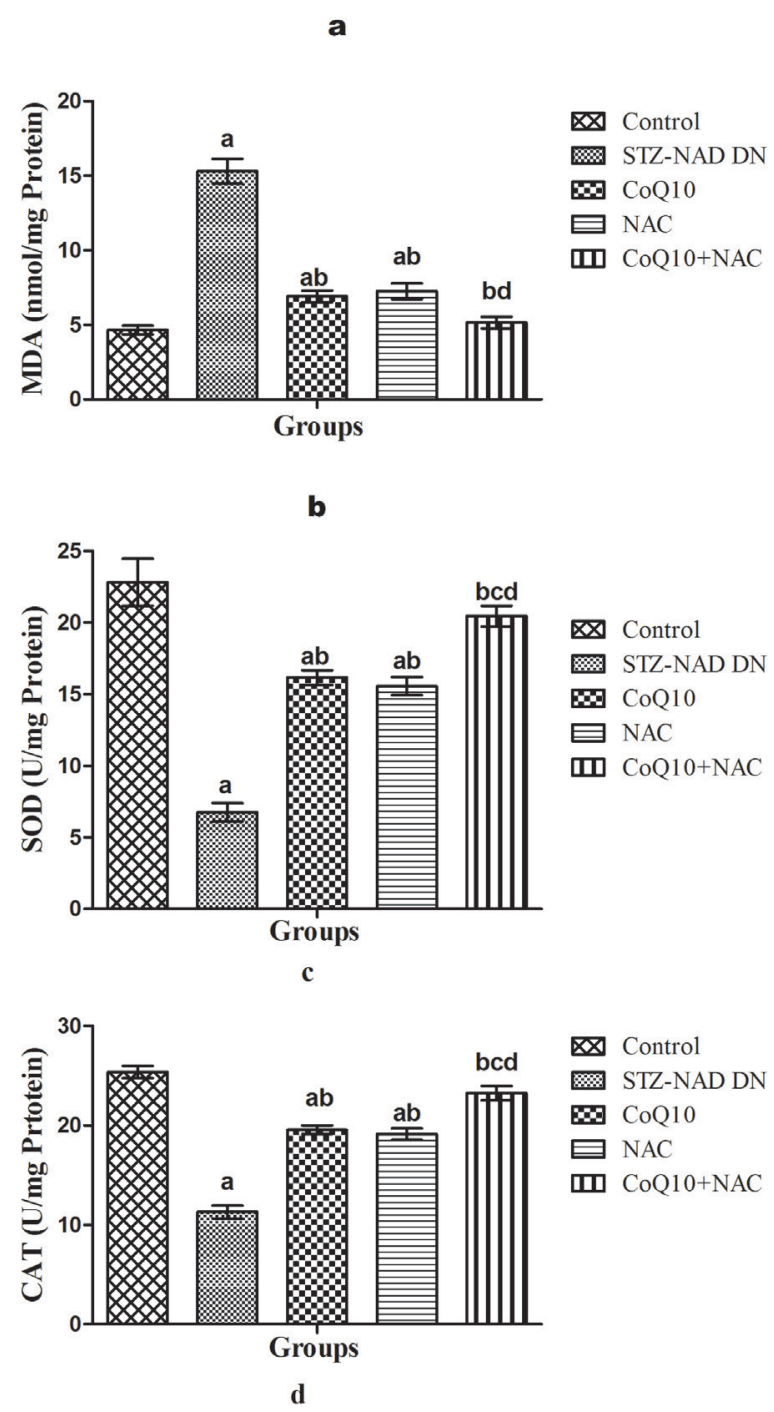

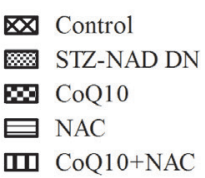

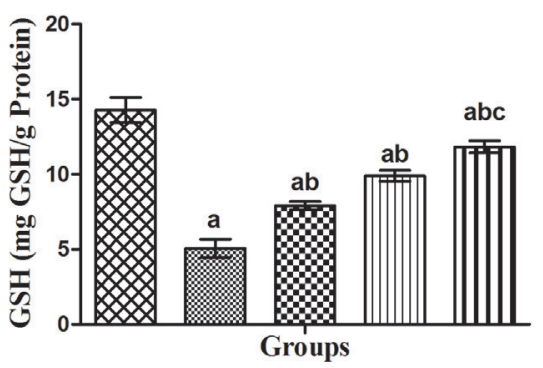

Control

STZ-NAD DN

EO CoQ10

एس $\mathrm{NAC}$

سा CoQ10+NAC

Figure 3. Effect of CoQ10, NAC or their combination on renal OS and anti-oxidant markers a) MDA, b) SOD, c) CAT and d) GSH.

structural and functional abnormalities in the kidney along with the increased OS. These observations concerned with DN were consistent with previous findings (Garjani et al., 2011; Maheshwari et al., 2014). STZ-NAD induced renal damage was revealed from increased MDA level, reduced SOD and CAT activities and decreased GSH content in renal tissue. Also, renal MPO activity and nitrite content were 
Table 4. Effect of CoQ10, NAC or their combination on MPO activity and NO in renal tissue.

\begin{tabular}{|c|c|c|}
\hline Groups & $\begin{array}{c}\text { MPO } \\
\text { (U/g Tissue) }\end{array}$ & $\begin{array}{c}\text { NO } \\
(\boldsymbol{\mu m o l} / \mathbf{g} \text { Tissue) }\end{array}$ \\
\hline Control & $8.00 \pm 0.718$ & $4.38 \pm 0.567$ \\
\hline STZ-NAD DN & $27.28 \pm 0.871^{\mathrm{a}}$ & $12.45 \pm 0.776^{\mathrm{a}}$ \\
\hline CoQ10 $(10 \mathrm{mg} / \mathrm{kg})$ & $16.63 \pm 0.639^{\mathrm{ab}}$ & $6.36 \pm 0.355^{\mathrm{b}}$ \\
\hline NAC $(300 \mathrm{mg} / \mathrm{kg})$ & $17.27 \pm 0.556^{\mathrm{ab}}$ & $7.23 \pm 0.480^{\mathrm{ab}}$ \\
\hline CoQ10 $+\mathrm{NAC}$ & $13.07 \pm 0.582^{\mathrm{abcd}}$ & $4.68 \pm 0.425^{\mathrm{bd}}$ \\
\hline
\end{tabular}

Values are expressed as mean $\pm S E M ; n=6$. One-way ANOVA followed by Tukey's multiple comparison test. ${ }^{a} p<0.05$ as compared to the normal control group, ${ }^{b} p<0.05$ as compared to the diabetic control group, ${ }^{c} p<0.05$ as compared to diabetic CoQ10 alone treated group and ${ }^{d} p<0.05$ as compared to diabetic NAC alone treated group.

increased significantly in the rats with DN. On the other hand, the treatment of rats with DN by the antioxidants CoQ10 or NAC improved the renal parameters with restriction of hyperglycaemia and restoration of anti-oxidant enzymes. Especially when the combined treatment was given, more beneficial outcomes were observed.

DN is rapidly becoming the major cause of the ESRD globally. Chronic hyperglycaemia is known to play a key role in all the diabetic complications including DN. Also, persistent hyperglycaemia is linked to the early and sustained OS generation during DM. OS and uncontrolled hyperglycaemia together play a decisive role in the pathogenesis of tubuloglomerular abnormalities (Mahajan et al., 2019).

Thus, one of the different strategies to alleviate DM or its complications is by using antioxidants. Various endogenous or exogenous antioxidants were found useful in diabetic complication including DN due to their ability to scavenge the free radicals and modulate various signalling pathways to restore the renal function. Antioxidants like vitamin $E$ and C, CoQ10, NAC, a-lipoic acid, taurine and others are found effective against oxidative cell damage (Ahmadi et al., 2013; Jemai et al., 2009). Therefore, antioxidants could be used effectively as a complementary therapy in OS induced complications like DN. Recent animal and human studies have shown the beneficial effects of $\mathrm{CoQ} 10$ on elevated $\mathrm{HbA}_{1 C^{\prime}}$ urea, and creatinine in DM (Maheshwari et al., 2017; Zhang et al., 2019). Studies with NAC revealed its effectiveness against renal impairment associated with diabetes as indicated by a reduced urinary protein and urinary thiobarbituric acid reactive substances (Shimizu et al., 2005; Lee et al., 2016).

In the current research, a significant decline in body weight $\%$ in diabetic rats was noted. This reduction in body weight could be due to the excessive degradation of tissue proteins and increased muscle wasting. It was also evident that insulin deficiency contributes to the decreased protein synthesis and low serum total protein levels in diabetic rats (Rajkumar \& Govindarajulu, 1991).
The results of the study showed that in diabetic rats treated with CoQ10, NAC or their combination, body weight \% improved significantly probably due to the protective effect of these antioxidants in controlling muscle wasting, enhanced glucose uptake, reduced insulin resistance and inhibition of gluconeogenesis (Rajkumar et al., 1999; Amin et al., 2014; Midaoui et al., 2008).

The kidney to body weight ratio of the diabetic rats increased significantly due to renal hypertrophy, which is the key feature in initial alteration by DM. It was previously shown that overutilization of glucose, glycogen accumulation, and increased lipogenesis in the renal tissue during DM lead to kidney hypertrophy (Teoh et al., 2010; Mogensen, 1999). Treatment of diabetic rats with CoQ10, NAC or their combination effectively attenuated kidney hypertrophy.

Persistent hyperglycaemia has direct linkages the development of DN. Elevated blood glucose affects the endogenous antioxidant defense system and aggravates the development of DN through intracellular ROS, lipid peroxidation and leakage of urinary protein (Algenstaedt et al., 2003). In the current investigation, persistent hyperglycaemia was developed in diabetic rats until week 12 of the study period. Also, all the diabetic animals showed increased $\mathrm{HbA}_{1 \mathrm{c}}$. Diabetic animals treated with CoQ10, NAC or their combination for 8 weeks restricted hyperglycaemia and $\mathrm{HbA}_{1 \mathrm{c}}$.

Studies showed that patients with T2DM often have a deficiency of CoQ10 as marked by considerably lower levels of CoQ10 in plasma in comparison to healthy individuals, which in turn impair body's defense against hyperglycaemia induced OS (Ates et al., 2013; Hasegawa et al., 2005; Sourris et al., 2012; Mezawa et al., 2012). Mezawa et al showed the beneficial effects of $\mathrm{CoQ} 10$ in increasing insulin production and/or insulin secretion. Additionally, CoQ10 might attenuate OS in mitochondria and improve the functioning of $\beta$-cell along with enhanced insulin sensitivity (Anwar et al., 2014). Consequently, exogenous administration of CoQ10 could potentially attenuate mitochondrial dysfunction induced by OS, thus improving glycaemic control in T2DM (Alam et al., 2014).

Several clinical and experimental studies revealed the beneficial effects of NAC against insulin resistance and associated complications. In most of these studies, NAC reduced hyperglycaemia maybe by improving insulin sensitivity and enhancing peripheral glucose uptake by its antioxidant properties (Ammon et al., 1992; Ho et al., 1999). In this study, oral administration of NAC (300 mg/ $\mathrm{kg}$, p.o.) to the diabetic rats restricted hyperglycaemia than the diabetic control group but didn't reduce blood glucose levels in comparison to the observations from similar group at week 0 . Thus, the combined treatment employed herein may have a synergistic effect of CoQ10 and NAC in reducing hyperglycaemia and $\mathrm{HbA}_{1 c^{\circ}}$

It was shown that in DM, excessive excretion of total protein and albumin in urine leading to their reduced serum levels 
contributed to the pathogenesis of DN (Roy et al., 2010). CoQ10, NAC or their combination treatment restored serum total protein and albumin by preventing their urinary excretion. The combination treatment with CoQ10 and NAC in diabetic rats was found more effective than CoQ10 or NAC alone in restoration of serum total proteins and albumin.

The most common characteristics in the development of DN are the elevated serum creatinine $(\mathrm{SCr})$ and declined creatinine clearance (Ccr) (Dabla, 2010). The present study showed the significant elevation in the $\mathrm{SCr}$, and decline in $\mathrm{CCr}$ in diabetic groups than normal rats pointing to the declined renal function and development of DN. However, diabetic rats that received CoQ10 (10 mg/kg), NAC (300 mg/kg) or their combination decreased $\mathrm{SCr}$ and improved $\mathrm{CCr}$, particularly in the animals treated with the combination of the antioxidants. In addition to the chronic hyperglycaemia, polyuria is another feature of DM arising from osmotic diuresis. The significant increment in the urine output ( $\mathrm{mL} /$ day) noted in this study may be due to glucosuria associated osmotic diuresis. In this study, severe renal impairment in the diabetic rats was identified by substantial rise in the urinary total proteins and UAER. Decreased serum albumin and increased UAER in diabetic complications were linked to the rapid progression of renal disease.

Earlier reports demonstrated that DM induced renal dysfunction is associated with a gradual reduction in serum albumin level. Albuminuria, resulting from damage to the glycosaminoglycans in the basement membrane and increased pore size may be linked to the decreased serum albumin (Mora-Fernández et al., 2014; Haraldsson \& Sörensson, 2004). Consequently, the reduction of proteinuria could be beneficial for improving renal function and to prevent the progression of DN towards ESRD (Oktem et al., 2006). Combined oral administration of CoQ10 and NAC to the diabetic rats was found effective in reversing the urinary protein loss declining UAER.

OS is the key element in the development and progression of DN and associated renal injury. Diabetic state is believed to induce OS by an imbalance between the normal antioxidant defense and elevated ROS production. It has been shown that antioxidant treatment in DN improves renal function by directly acting against oxidative tissue damage (Mahajan et al., 2019). Moreover, exogenous administration of antioxidants restricts the progression of DN by effective inhibition of ROS and scavenging the preformed intracellular ROS (Agrawal \& Sadhukhan, 2015).

We noted impaired oxidative stability in diabetic rats as indicated by the elevated MDA level and reduced activity of antioxidant enzymes SOD and CAT. It is believed that during DM hyperglycaemia-induced OS increases lipid peroxidation (Idris et al., 2001). MDA, the product of lipid peroxidation is responsible for the cellular injury. As an aldehyde, MDA links sugar and protein to form glycated proteins. Such structural and functional abnormalities in proteins might be responsible for diabetic complications. Thus, increased levels of $\mathrm{HbA}_{1 c}$ may have some linkages with increased lipid peroxidation (Krhač \& Lovrenčić, 2019). There was a considerable decrease in lipid peroxidation and an elevation in SOD, CAT activity, along with increased GSH content in the renal tissue of rats treated orally with a combination of CoQ10 and NAC.

Studies correlated MPO and DM with a positive relationship between the raised MPO activity and the pathogenesis of DN (Rovira-Llopis et al., 2013). The MPO-hydrogen, a peroxidechloride system, leads to a variety of chlorinated protein and lipid adducts that may cause dysfunction in the different compartments of the kidney (Prabhakar, 2004). In the present study, oral combination therapy of diabetic rats with CoQ10 and NAC significantly reduced renal MPO activity in the renal tissue than the CoQ10 and NAC alone treated rats.

$\mathrm{NO}$ is implicated in pathogenesis of DN since it modulates renal structure and function. In diabetes, abnormal NO production is linked to the progression of the kidney damage. Studies showed that most of the cytotoxicity attributed to NO is due to peroxynitrite, produced from the reaction between NO and the superoxide anion (Stamler et al., 1992; Kisic et al., 2016). In the present study, there was a significant elevation in nitrite content in the renal tissue of diabetic rats as compared to the normal rats. However, the diabetic rats treated with a combination of $\mathrm{CoQ} 10$ and NAC effectively reduced renal nitrite levels.

In conclusion, the results presented in this study provide valuable information supporting that treatment with antioxidants might prevent or delay the renal damage associated with DN. Oral administration of antioxidants, that is, CoQ10 and NAC possesses a significant nephroprotective effect against STZ-NAD induced DN. A combination therapy with CoQ10 and NAC is more promising as it improves renal function and reduces $O S$ in the rats subjected to DN. Nephroprotective effect shown by the combination treatment in this study may be attributed to hypoglycaemic and antioxidant properties of these compounds that attenuated OS and enhanced renal function in diabetic rats. Finally, it was concluded that the combined administration of CoQ10 with NAC might attenuate or delay the progression of DN.

\section{ABBREVIATIONS}

AGEs: Advanced glycation end products

ANOVA: Analysis of variance

ATP: Adenosine triphosphate

BUN: Blood urea nitrogen

CAT: Catalase

CoQ10: Coenzyme Q10

DM: Diabetes mellitus

DN: Diabetic nephropathy

GLUT2: Glucose transporter 2

GSH: Reduced glutathione

h: Hour 
$\mathbf{H b A}_{1 c}$ : Glycated haemoglobin

i.p.: Intraperitoneal

MDA: Malondialdehyde

MPO: Myeloperoxidase

NAC: N-acetylcysteine

NAD: Nicotinamide

NIDDM: Non-insulin-dependent diabetes mellitus

NO: Nitric oxide
OS: Oxidative stress

p.o.: Per oral

PKC: Protein kinase $C$

ROS: Reactive oxygen species

SEM: Standard error of the mean

SOD: Superoxide dismutase

STZ: streptozotocin

T2DM: Type-2 diabetes mellitus

\section{References}

[1] Agarwal N, Sadhukhan P. Therapeutic Insights against Oxidative Stress Induced Diabetic Nephropathy: A Review. J Autoimmune Disord. 2015; 1: 1-17.

[2] Ahmad A, Mondello S, Di Paola R et al. Protective effect of apocynin, a NADPH-oxidase inhibitor, against contrastinduced nephropathy in the diabetic rats: A comparison with n-acetylcysteine. Eur J Pharmacol. 2012; 674: 397-406.

[3] Ahmadi A, Mazooji N, Roozbeh J, Mazloom Z, Hasanzade J. Effect of alpha-lipoic acid and vitamin $E$ supplementation on oxidative stress, inflammation, and malnutrition in hemodialysis patients. Iran J Kidney Dis. 2013; 7: 461-467.

[4] Ahmadvand H. Effects of coenzyme Q10 on hemoglobin A1C, serum urea and creatinine in alloxan-induced Type 1 diabetic rats. Iran J Pharmacol Ther. 2012; 11: 64-67.

[5] Alam M, Rahman M. Mitochondrial dysfunction in obesity: Potential benefit and mechanism of co-enzyme Q10 supplementation in metabolic syndrome. J Diabetes Metab Disord. 2014; 13: 1-11.

[6] Algenstaedt P, Schaefer C, Biermann T, et al. Microvascular Alterations in Diabetic Mice Correlate with Level of Hyperglycemia. Diabetes. 2003; 52: 542-549.

[7] Amin M, Asaad G, Abdel Salam R, El-Abhar H, Arbid M. Novel CoQ10 Antidiabetic Mechanisms Underlie Its Positive Effect: Modulation of Insulin and Adiponectine Receptors, Tyrosine Kinase, PI3K, Glucose Transporters, sRAGE and Visfatin in Insulin Resistant/Diabetic Rats. PLoS One. 2014; 9: e89169.

[8] Ammon HP, Muller PH, Eggstein M, Wintermantel C, Aigner $B$, Safayhi $H$, et al. Increase in glucose consumption by acetylcysteine during hyperglycemic clamp. A study with healthy volunteers. Arzneimittelforsch. 1992; 42: 642-645.

[9] Anwar M,AbdEl-Moniem M, Megahed H,EL-Toukhy S, Mohammed $\mathrm{N}$, Youness E. Effect of Coenzyme Q10 Supplementation on Markers of Oxidative Stress in Streptozotocin Induced Diabetic Rats. Journal of Applied Pharmaceutical Science. 2014; 4: 9-15.

[10] Ates O, Bilen H, Keles S, Alp H, Keles M, et al. Plasma coenzyme Q10 levels in type 2 diabetic patients with retinopathy. Int J Ophthalmol. 2013; 6: 675-679.

[11] Badole SL, Bagul PP, Mahamuni SP et al. Oral L-glutamine increases active GLP-1 (7-36) amide secretion and improves glycemic control in streptozotocin-nicotinamide induced diabetic rats. ChemBiol Interact. 2013; 203: 530-541.

[12] Brownlee M. Biochemistry and molecular cell biology of diabetic complications. Nature. 2001; 414: 813-20.
[13] Dabla PK. Renal function in diabetic nephropathy. World J Diabetes. 2010; 1: 48-56.

[14] Garjani A, Andalib S, Biabani S, Soraya H, Doustar Y, et al. Combined atorvastatin and coenzyme Q10 improve the left ventricular function in isoproterenol-induced heart failure in rat. Eur J Pharmacol. 2011; 666: 135-141.

[15] Geraldes P, Hiraoka-Yamamoto J, Matsumoto M, Clermont A, Leitges $M$, et al. Activation of PKC-delta and SHP-1 by hyperglycemia causes vascular cell apoptosis and diabetic retinopathy. Nat Med. 2009; 15: 1298-1306.

[16] Ghasemi A, Khalifi S, Jedi S. Streptozotocin-nicotinamide induced rat model of type 2 diabetes. Acta Physiol Hung. 2014; 101:408-420.

[17] Haber CA, Lam TK, Yu Z et al. N-acetylcysteine and taurine prevent hyperglycemia-induced insulin resistance in vivo: possible role of oxidative stress. Am J Physiol Endocrinol Metab. 2003; 285: E744E753.

[18] Haraldsson B, Sörensson J. Why do we not all have proteinuria? An update of our current understanding of the glomerular barrier. News Physiol Sci. 2004; 19: 7-10.

[19] Hasegawa G, Yamamoto Y, Zhi J, Tanino Y, Yamasaki M, et al. Daily profile of plasma \%CoQ10 level, a biomarker of oxidative stress, in patients with diabetes manifesting postprandial hyperglycaemia. Acta Diabetol. 2005; 42: 179-181.

[20] Ho E, Chen G, Bray TM. Supplementation of N-acetylcysteine inhibits NF-kappaB activation and protects against alloxaninduced diabetes in CD-1 mice. FASEB J. 1999; 13: 1845-1854.

[21] Hodgson JM, Watts GF, Playford DA, Burke V, Croft KD. Coenzyme Q10 improves blood pressure and glycaemic control: A controlled trial in subjects with type 2 diabetes. Eur J Clin Nutr. 2002; 56: 1137-1142.

[22] Idris I, Gray S, Donnelly R. Protein kinase C activation: isozymespecific effects on metabolism and cardiovascular complications in diabetes. Diabetologia. 2001; 44: 659-673.

[23] Jemai H, El Feki A, Sayadi S. Antidiabetic and antioxidant effects of hydroxytyrosol and oleuropein from olive leaves in alloxandiabetic rats. J Agric Food Chem. 2009; 57: 8798-8804.

[24] Kisic B, Miric D, Dragojevic I, Rasic J, Popovic L. Role of Myeloperoxidase in Patients with Chronic Kidney Disease. Oxid Med Cell Longev. 2016; 1-10.

[25] Kohei KA. Pathophysiology of type 2 diabetes and its treatment policy. JMAJ. 2010; 53: 41-46.

[26] Krhač M, Lovrenčić MV. Update on biomarkers of glycemic control. World J Diabetes. 2019; 10: 1-15. 
[27] Lee ES, Kim HM, Kang JS, Lee EY, et al. Oleanolic acid and $\mathrm{N}$-acetylcysteine ameliorate diabetic nephropathy through reduction of oxidative stress and endoplasmic reticulum stress in a type 2 diabetic rat model. Nephrol Dial Transplant. 2016; 3: 391-400.

[28] Mahajan MS, Gulecha VS, Upaganlawar AB, Upasani CD. Antioxidant Defense in Diabetic Nephropathy In: Uddin $S$ \& Upaganlawar AB: Oxidative Stress and Antioxidant Defense: Biomedical value in Health and Diseases, 1 st ed. New York: Nova; 2019.

[29] Maheshwari R, Balaraman R, Sen AK, Shukla D, Seth A. Effect of concomitant administration of coenzyme Q10 with sitagliptin on experimentally induced diabetic nephropathy in rats. Renal Failure. 2017; 39: 130-139.

[30] Maheshwari RA, Balaraman R, Sen AK, Seth AK. Effect of coenzyme Q10 alone and its combination with metformin on streptozotocin-nicotinamide-induced diabetic nephropathy in rats. Indian J Pharmacol. 2014; 46: 627-32.

[31] Maitra A. The Endocrine System. in: Kumar V, Abbas A, Fausto N, Aster J. Robbins and Cotran Pathologic Basis of Disease. 9th ed. Philadelphia: Elsevier Saunders; 2015.

[32] Masharani, U. Diabetes Mellitus and hypoglycemia. In: McPhee SJ, Papadakis MA, Tierney LM. Current medical diagnosis and treatment, 47th ed. New York: McGraw-Hill; 2008.

[33] Mezawa M, Takemoto M, Onishi S, Ishibashi R, Ishikawa T, Yamaga, $M$, et al. The reduced form of coenzyme Q10 improves glycemic control in patients with type 2 diabetes: an open label pilot study. Biofactors. 2012; 38: 416-421.

[34] Midaoui A, Ismael M, Lu H, Fantus I, de Champlain J, Couture R. Comparative effects of $\mathrm{N}$-acetyl-L-cysteineand ramipril on arterial hypertension, insulin resistance, andoxidative stress in chronically glucose-fed rats. Can J Physiol Pharmacol. 2008; 86: 752-60

[35] Mogensen CE. Microalbuminuria, blood pressure and diabetic renal disease. Origin and development of ideas. Diabetologia. 1999; 42: 263-285.

[36] Mogensen CE, Christensen CK, Vittinghus E. The stages in diabetic renal disease. With emphasis on the stage of incipient diabetic nephropathy. Diabetes. 1983; 32: 64-78.

[37] Mora-Fernández C, Domínguez-Pimentel V, de Fuentes MM, et al. Diabetic kidney disease: from physiology to therapeutics. J Physiol. 2014; 592: 3997-4012.

[38] Odetti P, Pesce C, Traverso N, Menini S, Maineri EP, et al. Comparative trial of $\mathrm{N}$-acetyl-cysteine, taurine, and oxerutin on skin and kidney damage in long-term experimental diabetes. Diabetes. 2003; 52: 499-505.

[39] Oktem F, Ozguner F, Yilmaz HR, et al. Melatonin reduces urinary excretion of $\mathrm{N}$-acetyl-beta-D-glucosaminidase, albumin and renal oxidative markers in diabetic rats. Clin Exp Pharmacol Physiol. 2006; 33: 95-101.

[40] Pal PB, Sinha K, Sil PC. Mangiferin attenuates diabetic nephropathy by inhibiting oxidative stress mediated signaling cascade, TNFa related and mitochondrial dependent apoptotic pathways in streptozotocin-induced diabetic rats. PLoS One. 2014; 9: e107220.
[41] Prabhakar SS. Role of nitric oxide in diabetic nephropathy. Semin Nephrol. 2004; 24: 333-344.

[42] Rajkumar L, Govindarajulu P. Increased degradation of dermal collagen in diabetic rats. Indian J Exp Bio. 1991; 29: 1081-1083.

[43] Rajkumar V, Ragatzki P, Sima A, Levy J. Enhanced platelet aggregation, high homocysteine level, and microvascular disease in diabetic muscle infaractions. Endocrine. 1999; 11: 57-60.

[44] Rosenfeldt FL, Haas SJ, Krum H. Coenzyme Q10 in the treatment of hypertension: a meta-analysis of the clinical trials. J Hum Hypertens. 2007; 21: 297-306.

[45] Rovira-Llopis S, Rocha M, Victor $M$ et al. Is myeloperoxidase a key component in the ROS-induced vascular damage related to nephropathy in type 2 diabetes?" ARS. 2013; 19: 1452-1458.

[46] Roy S, Trudeau K, Roy S, Behl Y, Dhar S, Chronopoulos A. New insights into hyperglycemia-induced molecular changes in microvascular cells. J Dent Res. 2010; 89: 116-128.

[47] Schena FP and Gesualdo L. Pathogenetic mechanisms of diabetic nephropathy. J. Am. Soc. Nephrol. 2010; 16: 30-33.

[48] Shimizu MH, Coimbra TM, de Araujo M et al. N-acetylcysteine attenuates the progression of chronic renal failure.Kidney Int. 2005; 68: 2208-2217.

[49] Sourris K, Harcourt B, Tang P, Morley A, Huynh K, et al. Ubiquinone (coenzyme Q10) prevents renal mitochondrial dysfunction in an experimental model of type 2 diabetes. Free Radic Biol Med. 2012; 52: 716-723.

[50] Stamler JS, Singel DJ, Loscalzo J. Biochemistry of nitric oxide and its redox-activated forms. Science. 1992; 258: 1898-902.

[51] Teoh SL, Abdulatiff A, Das S. Histological changes in the kidneys of experimental diabetic rats fed with Momordicalcharantia (bitter gourd) extract. Rom J MorpholEmbryol. 2010; 51: 91-5.

[52] Vasavada N and Agarwal R. Role of Oxidative Stress in Diabetic Nephropathy. Adv Chronic Kidney Dis. 2005; 12: 146-154.

[53] Vora JP and Ibrahim HAA. Clinical manifestations and natural history of diabetic nephropathy. In: Johnson R and Feehally J: Comprehensive clinical nephrology, 2nd ed. Edinburgh: Mosby; 2003.

[54] Zhang X, Shi Z, Liu Q, Quan H, Cheng X. Effects of coenzyme Q10 intervention on diabetic kidney disease. Medicine. 2019; 98: 1-18. 